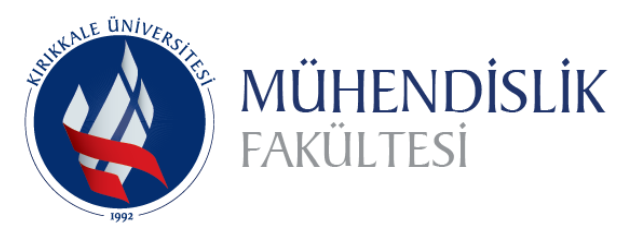

Uluslararası Mühendislik
Araştırma ve Geliştirme Dergisi
International Journal of
Engineering Research and
Development

Cilt/Volume:14 Sayı/Issue:1 Ocak/January 2022

Araştırma Makalesi / Research Article

\title{
Kasis Profilinin Pasif Süspansiyon Sistemli Çeyrek Araba Modeli Kullanılarak Sürüş Konforuna Etkisi
}

\section{The Effect of Bump Profile on Driving Comfort Using a Quarter Car Model with Passive Suspension System}

\author{
Cihan Berke Cülcüloglu1 ${ }^{\text {iD }}$, M. Taylan Daş ${ }^{1}$ iD \\ Kırıkkale Üniversitesi, Mühendislik ve Mimarlık Fakültesi, Makine Mühendisliği Bölümü, 71305 Kırlkkale/TÜRKIYE
}

Başvuru/Received: 30/03/2021

Kabul / Accepted: 06/07/2021

Çevrimiçi Basım / Published Online: 31/01/2022

Son Versiyon/Final Version: 31/01/2022

\section{$\ddot{O} \mathbf{z}$}

Sürüş konforu, sürücü sağlığı ve araç sürüşünün daha güvenli olması için önemli bir faktördür. Yol profiline bağlı olarak araç içi vücut ivmelenmesi azaltılarak sürüş konforu arttırılabilir. Bu çalışma, bir kasis üzerinden geçen araç için sistem davranışını ve sürücü konforunu incelemiştir. Kasis üzerinden geçiş hızları $5-25 \mathrm{~km} / \mathrm{sa}$ arasında değerlendirilmiştir. Kasis uzunluğu TS 6283 standardına göre $3.6 \mathrm{~m}$ olarak alınmıştır. Kasis boyunun vücut ivmelenmesine etkisini karşılaştırmak için kasis uzunluğu $2.4 \mathrm{~m}$ olarak da alınmış, farklı kasis uzunlukları için sistem davranışı incelenmiştir. Hareket denklemleri; Newton-Euler metodu kullanılarak çıkartılmış, Matlab-Simulink’te blok diyagramları oluşturulup benzetim yapılmıştır. Karekök ortalaması [Root Mean Square (RMS)] metodu kullanılarak ivme değerlerine göre dikey eksende vücut ivmelenmesi ve yer değiştirmesi incelenmiştir.

\section{Anahtar Kelimeler}

"Çeyrek araba modeli, Pasif süspansiyon, Sürüş konforu”"

\begin{abstract}
Driving comfort is an important factor for driver health and safer driving. Depending on the road profile, driving comfort can be increased by reducing the in-vehicle body acceleration. This study examined system behavior and driver comfort for a vehicle passing over a bump. Passing speeds over the bump are evaluated between $5-25 \mathrm{~km} / \mathrm{h}$. The bump length is taken as $3,6 \mathrm{~m}$ according to TS 6283 standard. In order to compare the effect of bump length on body acceleration, the bump length was also taken as $2,4 \mathrm{~m}$, and system behavior for different bump lengths was examined. Equations of motion were derived by using Newton-Euler method, block diagrams were created and simulated in Matlab-Simulink. Body acceleration and displacement on the vertical axis were investigated according to acceleration values using the Root Mean Square (RMS) method.
\end{abstract}

Key Words

"The Quarter car model, Passive suspension, Drive Comfort" 


\section{Giris}

Araç süspansiyon sistemi, araç gövdesi ve tekerleklerin ayrımını sağlayan mekanik bir sistemdir. Süspansiyon sistemi; yay, amortisör ve bağlantı elemanlarından oluşur. Süspansiyon sistemi; yol tutuş, yük taşıma ve yolcu konforu olarak 3 ana kriter altında incelenir. Yol profilinin düzensizliği araç dinamiğini ve sürüş konforunu etkiler. İyi bir şekilde tasarlanmış süspansiyon, yol bozukluklarından kaynaklı titreşimleri en aza indirgeyerek sürücü için konfor sağlamalıdır, (Rajendiran ve ark., 2016), (Ahmed, 2015). Süspansiyon sistemi yol ile araç gövdesi arasındaki bağlantıyı yapar. Ani duruşlarda ve bozuk yollarda araç kontrolünü sürdürmeyi sağlarken sürüş konforunu da sağlamalıdır, (Florin ve ark., 2013). Temel anlamda sürüş konforunu sağlamak için dikey ivmelenmeyi en aza indirmeyi hedefler, (Salem ve Ayman, 2009). Süspansiyon sisteminin sürüş konforuna etkisi olduğu kadar sürücünün vücut sistemi ve bel ağrıs1 üzerinde de etkisi vardır, (Agharkakli ve ark., 2012). Literatürde birçok farklı parametre ve yöntemle çeyrek araba modeli üzerinde sürücü konforu için çalışmalar yapılmışıı, (Phalke ve Anirban, 2017). Genel olarak geçmiş çalışmalarda, çeyrek araba modeli için 5$25 \mathrm{~km} / \mathrm{sa} \mathrm{hız} \mathrm{aralığında} \mathrm{kasis} \mathrm{üzerinden} \mathrm{geçişte} \mathrm{araç} \mathrm{davranışları} \mathrm{incelenmiştir.} \mathrm{Hareket} \mathrm{denklemleri} \mathrm{Newton'un} \mathrm{ikinci} \mathrm{yasasına} \mathrm{uygun}$ olarak oluşturulmuştur. Matlab-Simulink’te blok diyagramlarla tasarlanan çeyrek araç modelinin dikey yöndeki yer değiştirme ve ivme grafikleri oluşturulmuştur. Karekök ortalaması metoduyla ivme grafikleri oluşturulmuştur, (Phalke ve Anirban, 2017), (Nagarkar ve ark., 2016), (Hassaan, 2014), (Hassaan, 2015). Genel olarak bu çalışmada kasis üzerinden geçişlerde farklı hız değerlerinin ve kasis uzunluğunun sürüş konforuna etkisi incelenmiştir.

\section{Matematiksel Modelleme}

Matematiksel modelleme veya hareket denklemlerinin çıkarımı bir sistemin en basit anlamda sistem davranışını ele alan analitik denklemlerin elde edilmesidir. Mekanik sistemlerde hareket denklemlerini elde etmek için Newton-Euler denklemleri veya enerji metodu olarak bilinen Lagrange yaklaşımı kullanılabilir. Bu çalışma kapsamında süspansiyon sistemi pasif olarak ele alınmış olup matematiksel modeli Newton yaklaşımıyla elde edilmiştir.

Günümüz araçlarında yaygın olarak kullanılan pasif süspansiyon sistemleri yol profili ve araç parametrelerine göre süspansiyon elemanlarından oluşur. Teknolojinin gelişmesiyle birlikte yarı aktif ve aktif süspansiyon sistemleri de kullanılmaya başlanmıştır. Bu sistemler elektronik kontrol ile her bir süspansiyon sistemini ayrı ayrı denetler ve hidrolik olarak dışarıdan bir kuvvet uygular. Yani farklı yol koşullarına ve rüzgârın oluşturduğu yanal kuvvetlere göre tepki verip süspansiyonu ayarlar.

$\mathrm{Bu}$ çalışmada kullanılan pasif süspansiyonlu çeyrek araba modeli Şekil 1(a)'da gösterilmiştir. Literatürde yaygın olarak kullanılmaktadır; çünkü modellenmesi ve hesaplanması basittir. Oluşturulan model iki serbestlik derecelidir: $\left(\mathrm{x}_{1}, \mathrm{x}_{2}\right)$. Giriş sinyali yani dış kuvvet olarak da tanımlanan yol profilinin araç üzerindeki etkisi (z) gösterilmiştir. Serbest kütleler arasına konumlandırılmış yay ve sönüm elemanı amortisörü oluşturur. $\mathrm{M}_{1}$ kütlesi ve yol profili arasında konumlandırılmış yay ve sönüm elemanı, lastik sertliği ve lastik içerisindeki havayı temsil etmektedir.

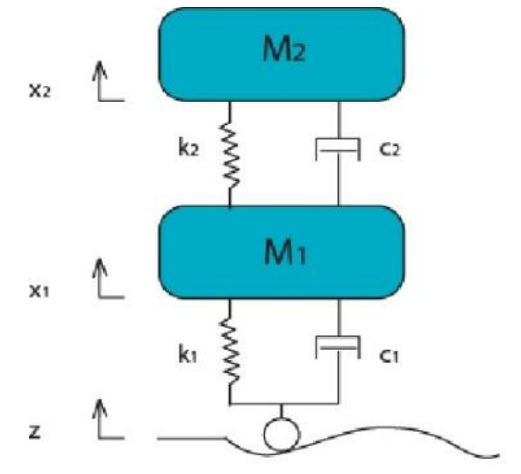

(a)

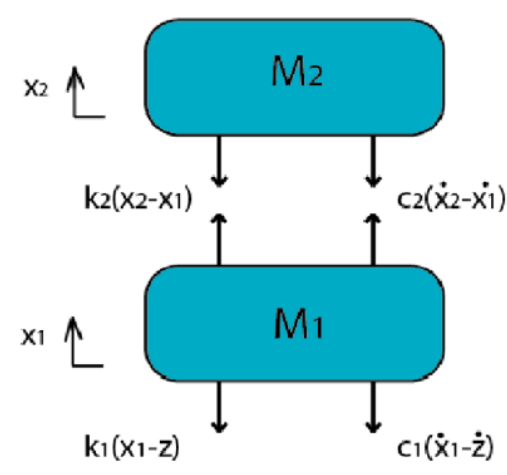

(b)

Şekil 1. (a) Çeyrek araba modeli, (b) Çeyrek araba modelinin serbest cisim diyagramı 
Şekil 1 (b)'de serbest cisim diyagramında gösterildiği gibi yay ve sönüm elemanlarının kütleler üzerindeki kuvvet etkileri oluşturulmuştur.

Hareket denklemlerini oluşturmak için $\mathrm{x}_{2}>\mathrm{x}_{1}>\mathrm{z}$ kabulü ile; denklem (1) ve (2) de gösterilmiştir.

$M_{2} \ddot{x_{2}}=-k_{2} \cdot\left(x_{2}-x_{1}\right)-c_{2} \cdot\left(\dot{x_{2}}-\dot{x_{1}}\right)$

$\mathbf{M}_{1} \ddot{\mathbf{x}_{1}}=\mathbf{k}_{2} \cdot\left(\mathbf{x}_{2}-\mathbf{x}_{1}\right)+\mathbf{c}_{2} \cdot\left(\dot{\mathbf{x}_{2}}-\dot{\mathbf{x}_{1}}\right)-\mathbf{k}_{1} \cdot\left(\mathbf{x}_{1}-\mathbf{z}\right)-\mathbf{c}_{1} \cdot\left(\dot{\mathbf{x}_{1}}-\dot{\mathbf{z}}\right)$

Tablo 1: Parametre değerleri, (Mahajan, 2016)

\begin{tabular}{ccc}
\hline Tanımlama & Parametre & Değer \\
\hline Süspansiyon ağırlı̆̆ı & $\mathrm{M}_{1}(\mathrm{~kg})$ & 28 \\
Araç gövdesinin ă̆ırlı̆̆ı & $\mathrm{M}_{2}(\mathrm{~kg})$ & 208 \\
Tekerlek yay katsayısı & $\mathrm{k}_{1}(\mathrm{~N} / \mathrm{m})$ & 127200 \\
Süspansiyon yay katsayısı & $\mathrm{k}_{2}(\mathrm{~N} / \mathrm{m})$ & 18709 \\
Süspansiyon sönüm katsayısı & $\mathrm{c}_{2}(\mathrm{Ns} / \mathrm{m})$ & 1300 \\
Tekerlek sönüm katsayısı & $\mathrm{c}_{1}(\mathrm{Ns} / \mathrm{m})$ & 10 \\
\hline
\end{tabular}

\subsection{Yol Profili Modeli}

Modellemedeki sistem üzerinde girdi sinyali olarak hız kesici tümsek Şekil 2'de şematik olarak gösterilmiştir. Türk Standartları Enstitüsünün TS 6283 standardına uygun şekilde aşağıdaki gibi modellenmiştir. Kasis tipleri, kısa, uzun ve düz tepeli olmak üzere 3 farklı kategoride sınıflandırılmaktadır. Bu çalışma kapsamında TS6283 standartı kapsamında boyu 3.6- 3.80 m olarak kullanılan uzun tümsek modeli ele alınmıştır. Avrupa birliği standartlarında kasis uzunluğu 3.7 - $4.25 \mathrm{~m}$ arasında kullanılmaktadır. (Arslan ve Hossainy,2020)

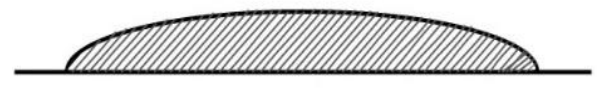

Şekil 2: Uzun kasis-Yol profili

$$
\begin{array}{ccc}
0<t<\frac{T}{2} & \text { için } & z=Z \sin \left(w_{z} t\right) \\
t>\frac{T}{2} & \text { için } & z=0 \\
\mathrm{Z}=0.1 \mathrm{~m} & \mathrm{~L}=3.6 \mathrm{~m}
\end{array}
$$

Yukarıdaki denklem için kasis yüksekliği Z, kasis genişliği L, kasis frekansı $\mathrm{w}_{\mathrm{z}}$ ve zaman t olarak tanımlıdır. Kasis frekansının hesaplanabilmesi için araba modelinin ne kadar sürede kasisten geçtiği, yani periyodu denklem (3) ile hesaplanabilir.

Kasis profili sinüsün bir fonksiyonu olarak modellenmiştir. Sinüs dalgasının bir tam periyodu göz önüne alındığında, sinüs dalgasının $0<t<\frac{T}{2}$ arasındaki yarım periyodu ile kasis geometrisi oluşturulabilir. $t>\frac{T}{2}$ olarak tanımlanan kısımda z değişkenini sıfıra götürerek kasis sonunda düz bir yol profili tanımlanabilir.

$T / 2=L /(V .1000 / 3600)$

Hesaplanan periyot ile;

$W_{Z}=\frac{2 \pi}{T}$

Denklem (4)'te frekans değeri hesaplanabilir.

Kasis uzunluğunun sürüş konforuna etkisini inceleyebilmek için, kasis boyunun kısaldığında araç davranışının karşılaştırılabilmesi için; $\mathrm{Z}=0.1 \mathrm{~m}$ ve $\mathrm{L}=2.4 \mathrm{~m}$ olarak alınıp tartışma kısmında değerlendirilmiştir. 


\section{Modelleme ve Blok Diyagramların Gösterimi}

\section{1. Çeyrek araba modeli}

Hareket denklemlerinin çözülebilmesi için Matlab-Simulink kullanılmıştır. Hareket denklemleri Şekil 3'te görüldüğü gibi blok diyagramlar olarak modellenmiştir. Giriş sinyali olarak yol profili sinüs dalgası şeklinde Şekil 4 'te gösterildiği gibi modellenip sistemin davranışı analiz edilmiştir. Parametreleri ve giriş sinyali girdisini kullanarak farklı araç hızları ve kasis uzunlukları için süspansiyon ve araç kütlesinin hareket grafikleri program üzerinde çizdirilebilir ve sistem davranışı incelenebilir.

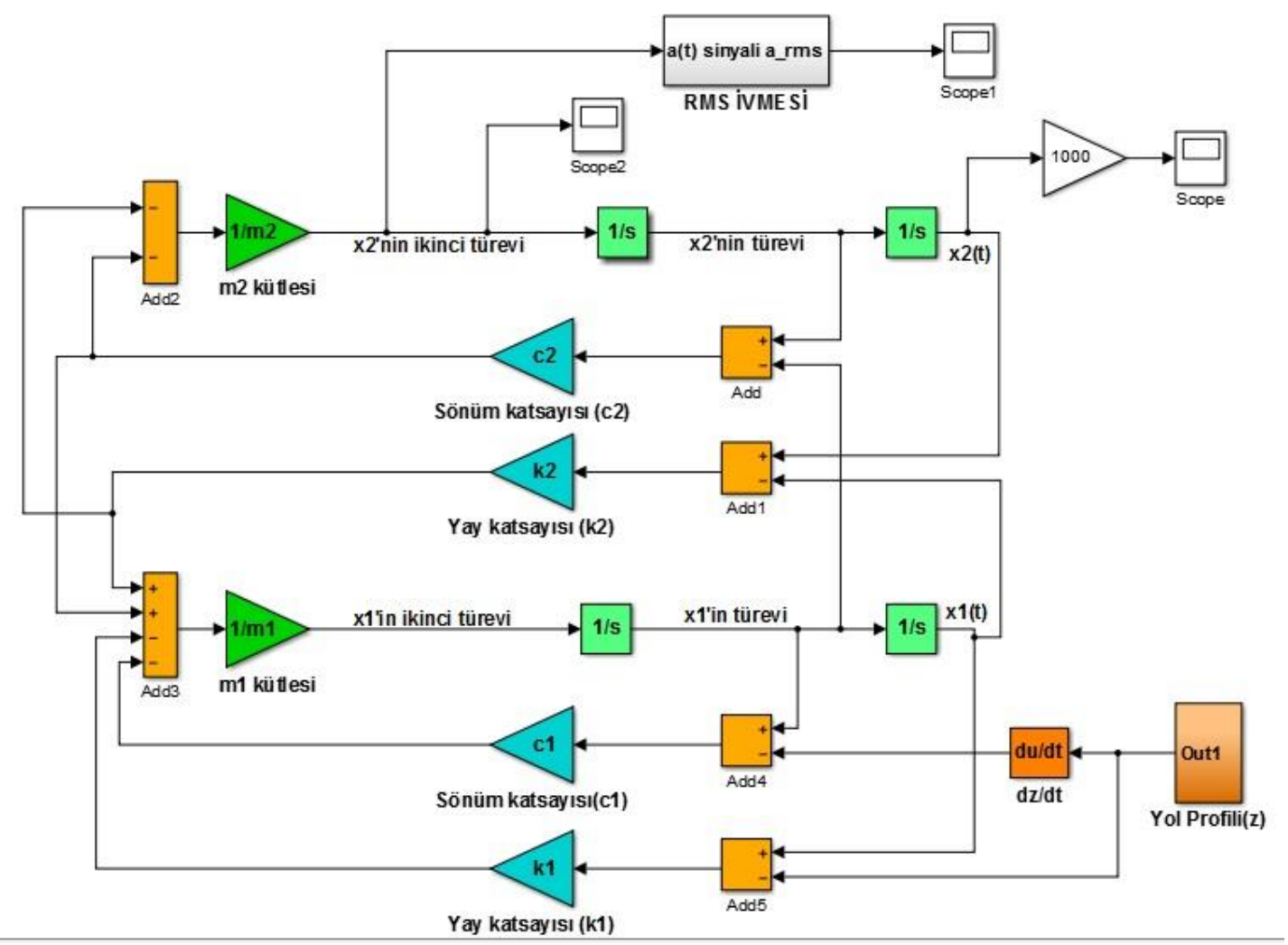

Şekil 3. Çeyrek araba blok diyagramı- Simulink modeli

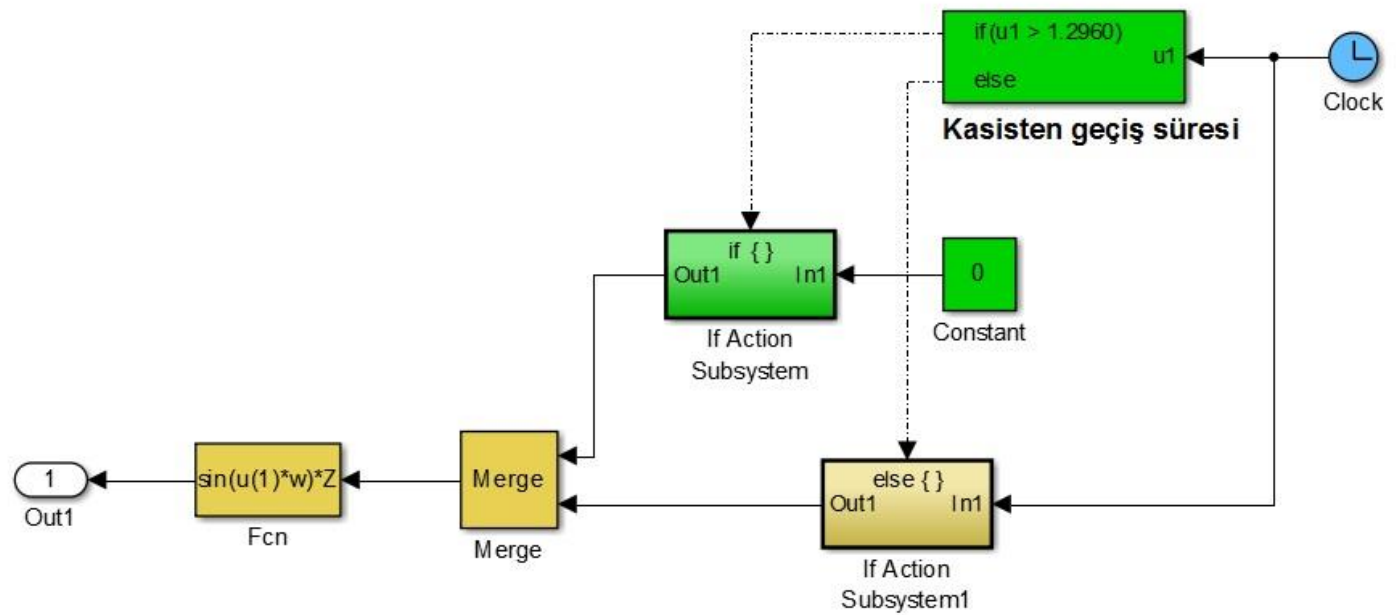

Şekil 4. Çeyrek araba blok diyagramı- Simulink modeli

Şekil 4'te aracın kasis üzerinden geçiş süresi v=10 km/sa ve L=3.6 m için $1.2960 \mathrm{~s}$ olarak gösterilmiştir. Bu süre yarım periyoda eşittir ve her hız değeri için değişkendir. 


\subsection{RMS İvmesi Modeli}

RMS metodu (Root Mean Square (karekök ortalaması)), sürüş konforunun belirli standartlar dahilinde irdelenebilmesi için kullanılan bir yöntemdir. Titreşim hareketi yapan sistemlerin farklı yönlerde salınım yapması pozitif ve negatif değerler ortaya çıkarır. Bu değerlerin ortalaması gerçeği yansıtmaz. Bu nedenle ISO-2631'de belirtilen aşağıdaki denklem yardımıyla her bir çıkış sinyalinin karelerinin karekök içerisinde ortalaması alınarak RMS değeri belirlenir. Denklem (5)'te verilen RMS ivme formülü Matlab-Simulink ile Şekil 5 'te görüldüğü gibi blok diyagram olarak modellenmiştir.

$a_{r m s}=\sqrt{\frac{1}{T} \int_{0}^{T} a_{w}^{2}(t) d t}$

ISO-2631 standardına göre $a_{r m s}$ ivmesi $0.315 \mathrm{~m} / \mathrm{s}^{2}$, den küçük olduğu durumlarda konforlu, $0.315<a_{r m s}<0.5$ az konforlu, $0.5<a_{r m s}<0.8$ biraz konforlu, $a_{r m s}>0.8$ durumunda konforsuz olarak belirtilmiştir, (Zoccali, 2018). Araç tasarımında kullanılan yükler arasında kasislerden kaynaklanan yükler de yer almaktadır, (Doğru, 2019). Ayrıca trafik kazalarına sebep olarak araç, yol ve sürücü durumlarının etkisinin sınıflandırılması da makine öğrenmesiyle çalışılmıştır, (Altın ve ark, 2021).

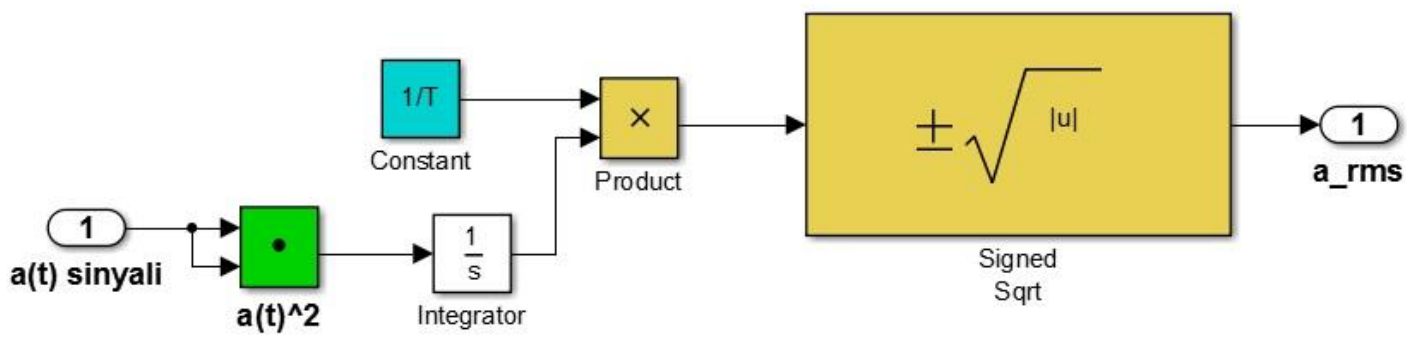

\section{Analiz Sonuçları Ve Tartışma}

Şekil 5. RMS ivmesi blok diyagramı-Simulink

Newton'un ikinci hareket yasasıyla çıkartılan hareket denklemleri ve Matlab-Simulink programında modellenen sistem için girdi sinyali olarak yol profilinin verildiği üst kısımlarda bahsedilmişti. Bu kısımda çeyrek araba modeli için bir kasis üzerinden belirli hızlarda $(\mathrm{v}=5-25 \mathrm{~km} / \mathrm{sa})$ geçişinde sistem davranışı, her bir hız değeri için M2 kütlesinin yer değiştirme grafikleri ve karekökleri ortalaması metodu ile vücut ivmesi grafikleri incelenmiştir. Standartta yer verilen $\mathrm{L}=3.6 \mathrm{~m}$ kasis boyunu, daha düşük bir kasis boyu ile karşılaştırmak için $\mathrm{L}=2.4 \mathrm{~m}$ kasis boyuna sahip bir kasisin sürüş konforuna ve vücut yer değiştirmesine etkisi de incelenmiştir.

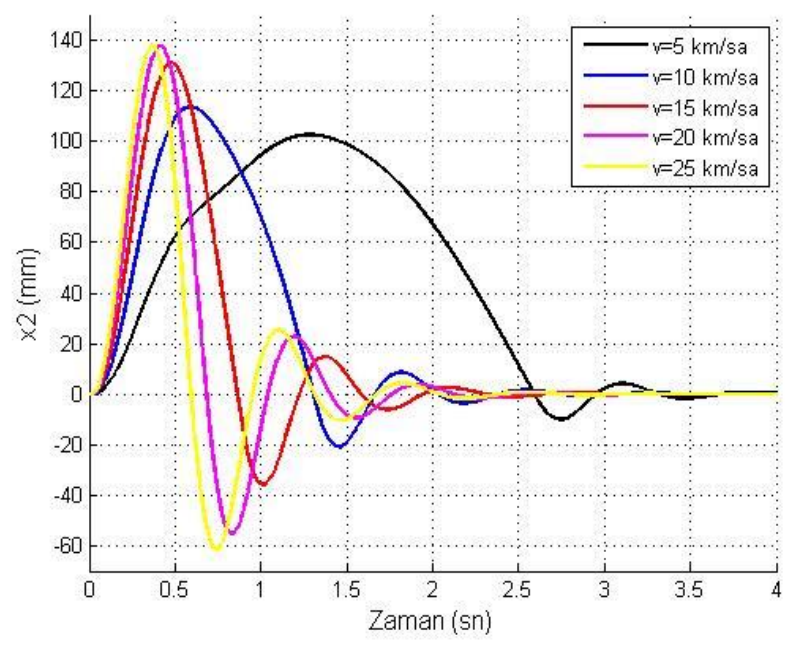

(a)

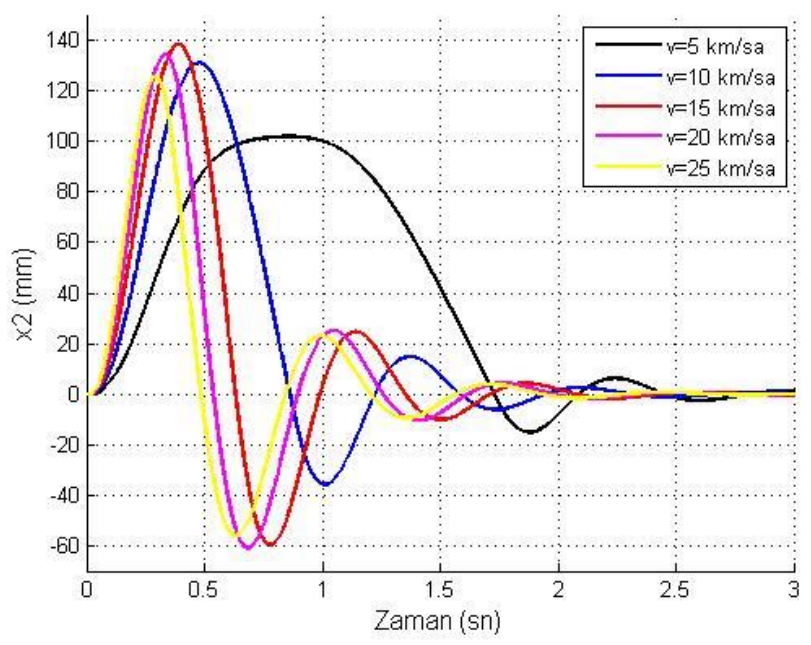

(b)

Şekil 6. (a) L=3.6 için M2’nin yer değiştirmesi, (b) L=2.4 için M2’nin yer değiştirmesi 


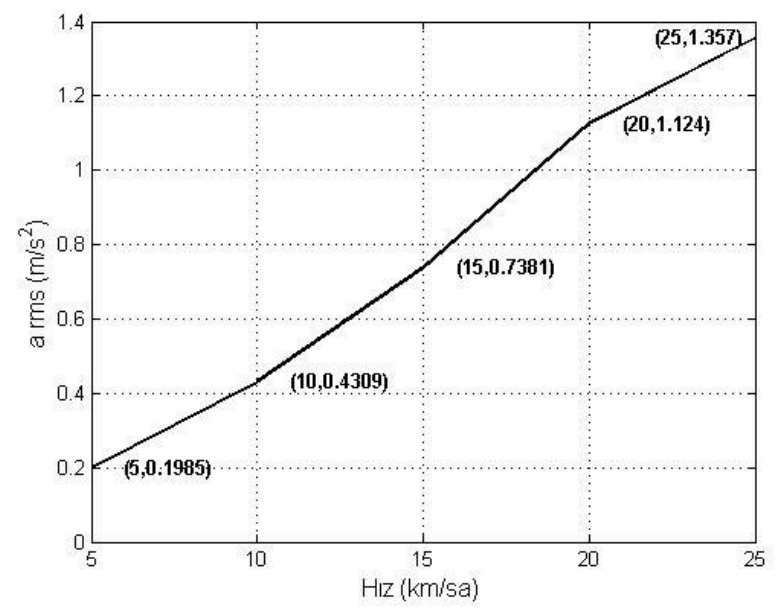

(a)

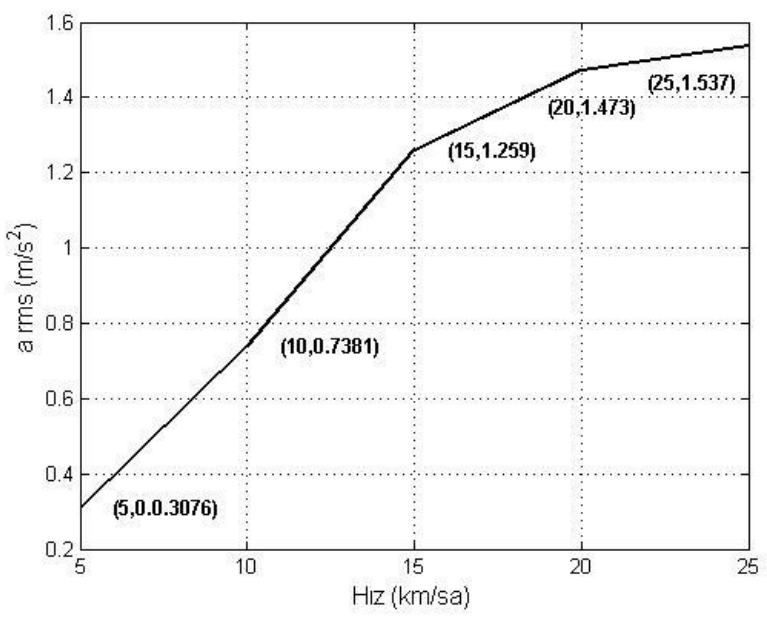

(b)

Şekil 7. (a) $\mathrm{L}=3.6$ için sürüş konforu $\mathrm{H}_{1 z}-\mathrm{a}_{\text {rms }}$ grafiği, (b) $\mathrm{L}=2.4$ için sürüş konforu Hız-a $\mathrm{a}_{\text {rms }}$ grafiği

Şekil 6.a ve Şekil 6.b'de görüldüğü üzere sistem davranışına bakıldığında hızın artmasıyla sistemin yerleşme zamanının azaldığı gözlemlenir. Bu durum, araç hızıyla yani tümsekten geçiş süresiyle ilişkilendirilebilir. Ancak araç hızının artmasıyla birlikte M2 kütlesinin maksimum ve minimum yer değiştirmesi de artmaktadır. Bu durum dikey eksen hareketinde daha düşük sürelerde daha yüksek bir hızlanma yaratacağı için ivmelenmeyi arttırır ve sürüş konforunu etkiler. Şekil 7.a ve Şekil 7.b'deki grafik 5-25 km/sa hız aralığında ve $\mathrm{L}=3.6 \mathrm{~m}, \mathrm{~L}=2.4 \mathrm{~m}$ kasis uzunlukları için $\mathrm{RMS}$ vücut ivmelenmesini göstermektedir. $\mathrm{a}_{\mathrm{rms}}$ ivmesinin $0.8 \mathrm{~m} / \mathrm{s}^{2}$ 'den küçük olduğu durumlar konforlu olarak nitelendirilir. Yani L=3.6 m için $16 \mathrm{~km} / \mathrm{sa}, \mathrm{L}=2.4 \mathrm{~m}$ için $10.6 \mathrm{~km} / \mathrm{sa}$ hızından daha düşük hızlar için sürüşün konforlu olduğu söylenebilir. Ayrıca kasis uzunluğunun azalmasıyla sürüş konforunun azaldığı da söylenebilir.

\section{Sonuç}

$\mathrm{Bu}$ çalışma, bir kasis üzerinden geçen pasif süspansiyon sistemli araç için sistem davranışını ve sürücü konforunu incelenmiştir. Hareket denklemleri Newton'un ikinci hareket yasasıyla çıkartılmış ve Matlab-Simulink’te sistem blok diyagramlarıyla modellenmiş, analiz edilmiş ve grafikleri çizdirilmiştir. Sürücü ivmelenmesi; karekök ortalaması metodu yardımıyla, kullanılan her bir hız değeri için hesaplanmış, grafiği çizdirilmiştir. Yol profilinden araç geçiş hızı arttıkça yerleşme zamanının azaldığı fakat vücut yer değiştirmesinin arttığı gözlemlenmiştir. Hesaplanan ortalama ivme değerlerinden L=3.6 m için $16 \mathrm{~km} / \mathrm{sa}, \mathrm{L}=2.4 \mathrm{~m}$ için $10.6 \mathrm{~km} / \mathrm{sa}$ 'ten daha düşük hızlarda konforlu olduğu belirtilmiştir. Kasis uzunluğunun azalmasıyla sürüş konforunun azaldığı belirlenmiştir.

\section{Kaynaklar}

Agharkakli, A., Sabet, G. S., \& Barouz, A. (2012). Simulation and analysis of passive and active suspension system using quarter car model for different road profile. International Journal of Engineering Trends and Technology, 3(5), 636-644.

Ahmed, A. E. N. S., Ali, A. S., Ghazaly, N. M., \& Abd el-Jaber, G. T. (2015). PID controller of active suspension system for a quarter car model. International Journal of Advances in Engineering \& Technology, 8(6), 899.

Arslan, T, Hossainy, M. (2020). Hız Tümseklerinin Araç ve Sürücüler/Yolcular Üzerindeki Etkilerinin Simülasyon Yöntemi ile Araştırılması. Osmaniye Korkut Ata Üniversitesi Fen Bilimleri Enstitüsü Dergisi, 3 (1) , 11-16 .

Doğru, M. H. (2019). Topology Optimization of Truck Chassis Under Multi Loading Conditions. El-Cezeri Journal of Science and Engineering, 6(3), 856-867.

Florin, A., Ioan-Cozmin, M. R., \& Liliana, P. (2013). Passive suspension modeling using MATLAB, quarter-car model, input signal step type. New technologies and products in machine manufacturing technologies, 258-263.

Hassaan, G. A. (2014). Car dynamics using quarter model and passive suspension, part I: effect of suspension damping and car speed. International Journal of Computer Techniques, 1(2), 1-9. 
Hassaan, G. A. (2015). Car dynamics using quarter model and passive suspension, Part II: a novel simple harmonic hump. Journal of Mechanical and Civil Engineering, 12(1), 93-100.

Mahajan, B. D., \& Divekar, A. A. (2016, May). Modeling and system identification of a quarter car suspension using Simulink. In 2016 IEEE International Conference on Recent Trends in Electronics, Information \& Communication Technology (RTEICT) (pp. 180-183). IEEE.

Nagarkar, M. P., Patil, G. J. V., \& Patil, R. N. Z. (2016). Optimization of nonlinear quarter car suspension-seat-driver model. Journal of advanced research, 7(6), 991-1007.

Phalke, T. P., \& Mitra, A. C. (2017). Analysis of Ride comfort and Road holding of Quarter car model by SIMULINK. Materials Today: Proceedings, 4(2), 2425-2430.

Rajendiran, S., \& Lakshmi, P. (2016). Simulation of PID and fuzzy logic controller for integrated seat suspension of a quarter car with driver model for different road profiles. Journal of Mechanical Science and Technology, 30(10), 4565-4570.

Salem, M. M. M., \& Aly, A. A. (2009). Fuzzy control of a quarter-car suspension system. World Academy of Science, Engineering and Technology, 53(5), 258-263.

Yavuz, A. A., Ergül, B., \& Aşık, E. G. (2021). Trafik Kazalarının Makine Öğrenmesi Yöntemleri Kullanılarak Değerlendirilmesi. International Journal of Engineering Research and Development, 13(1), 66-73.

Zoccali, P., Loprencipe, G., \& Lupascu, R. C. (2018). Acceleration measurements inside vehicles: Passengers' comfort mapping on railways. Measurement, 129, 489-498. 